\title{
Promoting health behaviors for preventing and controlling noncommunicable disease among North Korean defectors: A systematic review
}

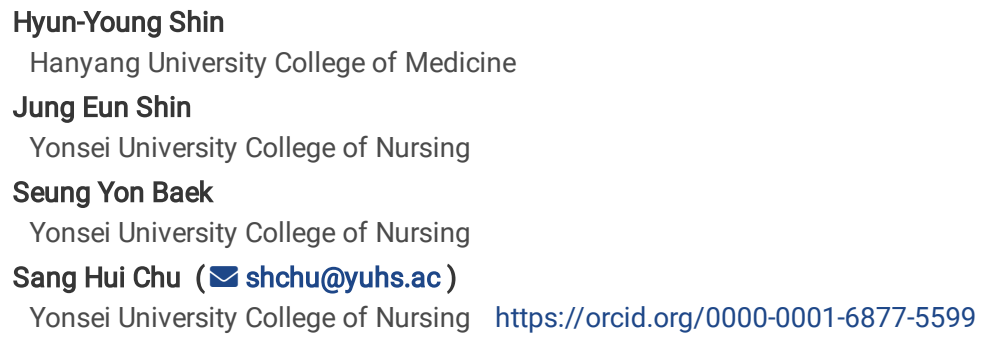

\section{Research article}

Keywords: North Korean defector, noncommunicable disease, health behavior

Posted Date: August 24th, 2020

DOl: https://doi.org/10.21203/rs.3.rs-56017/v1

License: () (1) This work is licensed under a Creative Commons Attribution 4.0 International License. Read Full License 


\section{Abstract}

Background: Recently, noncommunicable disease (NCDs) has emerged as a new major health issue in North Korea. However, studies on the prevalence of its risk factors and their related health behavior among North Koreans are lacking. The studies on North Korean defectors could help us in understating their awareness and health-promoting behavior as well as their vulnerabilities for metabolic diseases or NCDs under the current circumstances where South and North Korea have limited exchanges in the health and medical sectors. In this study, we reviewed the prevalence of risk factors or the health-promoting behavior related to NCD among North Korean defectors.

Methods: Search on the PubMed (MEDLINE), CINAHL, Cochrane Library, EMBASE, Korean Nursing Database, Korean Medical Database, KIHASA Repository, and RISS were performed. We included all study types reporting any type of risk factors or prevalence or health behaviors with the following inclusion criteria: (1) published in English or Korean; (2) NCD-related topic; and (3) age $\geq 18$ years. The data were extracted independently by the 2 authors

Results: Finally, 22 studies were included in the analysis: 18 quantitative study and 4 qualitative studies, and among the quantitative studies, 5 studies had comparisons with South Koreans. The number of participants ranged from 8-932 participants, and $54.6 \%-100 \%$ of them were women. The survey using the questionnaires was the most popular research method. Data from objective measurements, such as body weight, waist circumference, blood test, and urine analysis were used in a few studies. North Korean defectors were found to have higher smoking rates, drinking rates, and increased abdominal obesity caused by the lack of awareness and management of risk factors since they moved to South Korea.

Conclusions: In this study, we showed that there were not enough studies related to NCDs and health behaviors among North Korean defectors. Nevertheless, North Korean defectors were found to be more vulnerable to NCDs, including metabolic syndrome. It is necessary to improve the awareness of NCD and its risk factors among North Korean defectors. Efforts for promoting North Korean defectors' health behaviors by the government and related professional organizations are needed.

\section{Background}

Noncommunicable diseases (NCDs) have resulted in a significant public health burden. Globally, NCDs accounted for two-thirds of the total deaths, while communicable diseases and malnutrition accounted for only 25\% [1]. In high-income countries, better access to services for prevention, early diagnosis, and management of individuals with NCDs or at high risk of developing NCD have reduced deaths from cardiovascular disease (CVD). On the other hand, the situation in low- and middle- income countries is quite different. NCDs have a deteriorating impact in low- and middle-income countries with limited resources [2]. Thus, the prevention and control of NCDs by promoting healthy behavior, such as physical activity, balanced nutrition, regular check-up, and treatment adherence have been a priority for most countries with aged population. North Korea seems to be no exception. Since North Korea entered the aging society in 2004 , the proportion of the population aged 65 or older was expected to increase up to $9.5 \%$ in 2013 , entering an aged society in the year of 2030 [3]

Currently, North Korea is experiencing a "double burden" of diseases, with the increased burden of NCDs along with the previously existing health problems, malnutrition, and communicable diseases. Previously, in the 1990s, North Korea faced food and energy crisis owing to the severe collapse of economic conditions with the loss of trade links with its longstanding economic and trading partners in the socialist alliance [4]. The economic crisis soon disrupted the operation of North Korea's public health system, which advocates "free medical care." The situation further aggravated with a series of natural calamities (floods, typhoons, and droughts), which resulted in food insecurity and caused a serious public health situation (malnutrition), particularly among women and children [4]. There was a lack of medicine supply from other socialist countries; malnutrition also led to increased communicable diseases, such as tuberculosis (TB) and malaria.

Since the 1990s, the international aid to North Korea have been focused on improving maternal and children health (MCH), immunization, and controlling communicable diseases. With the help of the international community and non-governmental organizations (NGOs), the recent updated report on MCH in North Korea indicated some improvement, and immunization services have also been improved with rates $>90 \%$ for most antigens $[5,6]$. However, there is still much room to be improved in the $\mathrm{MCH}$ and communicable disease management.

In the midst of managing $\mathrm{MCH}$ and communicable diseases, NCDs have emerged as a new major health issue in North Korea [1, 7]. According to the report of the Institute for Health Metrics and Evaluation in 2015, the most common cause of death in North Korea was reported to be CVD accounting for $39 \%$ deaths, followed by the cerebrovascular disease with $21 \%$, the rates of which have increased by $18 \%$ and $17 \%$, respectively, compared to the last decade [8]. Tobacco usage, physical inactivity, excessive alcohol consumption, and unhealthy diets are the major risk factors for developing NCDs, thereby causing high blood pressure, hyperglycemia, elevated lipid profiles, and obesity [9]. However, studies on the prevalence of these risk factors and their related health behavior among North Koreans are still lacking. Therefore, research priorities need to be focused more on NCDs than on MCH and communicable diseases, which are currently the primary cause of morbidity and mortality in North Korea and is crucial for establishing an efficient health care policy for its prevention and control.

About 70 years of division between the two Korean countries have also led to differences in disease awareness, health behaviors, symptom expression, and an understanding of medical technology or information [10]. A higher prevalence of high blood pressure in men and low levels of high-density lipoprotein (HDL)cholesterol in women were observed in North Korean defectors compared to those in South Koreans [11]. Although North Korean defectors were relatively more likely to have increased visceral obesity and metabolic syndrome after a short period of settlement in South Korea [11, 12], there has been not much information on their health promotion behaviors. The studies on North Korean defectors could help us in understating their awareness and health-promoting behavior as well as their vulnerabilities for metabolic diseases or NCDs under the current circumstances where South and North Korea have limited exchanges in the health and medical sectors. 
In this study, we reviewed the prevalence of risk factors or the health-promoting behavior related to NCDs among North Korean defectors.

\section{Methods}

In accordance with good practice for systematic reviews, the study was conducted following the Preferred Reporting Items for Systematic Reviews and MetaAnalysis (PRISMA) guideline. Literature search on the PubMed (MEDLINE), CINAHL, Cochrane Library, EMBASE, Korean Nursing Database, Korean Medical Database, KIHASA Repository, and RISS were performed. Considering that there were not many studies conducted on North Korean defectors and no restrictions were imposed with regard to the year of publication at the initial search. Using the following key search terms ("North Korea* Refugee*" OR "Democratic people's republic of Korea" OR "North Korea* defector*"), we looked at all of the research done on North Korean defectors for finding out as much as possible

We included all study types reporting any type of risk factors or prevalence or health behaviors with the following inclusion criteria: (1) published in English or Korean; (2) NCD-related topic; and (3) age $\geq 18$ years. Dissertations, reviews, editorials, commentaries or unpublished articles were excluded. Study on psychology or psychiatry-related problems were also excluded. If multiple articles were published based on the same cohort study, the study containing the most complete or new information was selected.

Two authors (JES and SYB) screened all the articles by title and then by the abstract. Selected studies were then evaluated in greater depth based on the full text for the assessment of eligibility. If the reviewers disagreed, conflicts were resolved in consensus meetings with a third experienced researcher (SHC). Data were extracted independently by the 2 authors (JES and SYB) using a standard data extract form. We used a structured form for extracting standard information from each article, including general characteristics of participants, research purpose, comparators, measurements, and outcomes after several pre-testing, adjustments, and verifying the process for the appropriateness of the items contained and the consistency between the 2 different researchers. If different information were observed on the standard data extract form between the 2 researchers, consensus meetings with a third researcher were held repeatedly for confirming them. During the consensus meetings, individual observations were discussed, and the consensus was reached.

\section{Results}

The systematic literature search process is illustrated in figure 1. Our initial search yielded 820 studies. After removal of duplications, 493 studies were reviewed by title and then by the abstract. From this review process, 50 studies were included for a full-text review for the eligibility assessment. Twenty-eight articles were excluded owing to following reasons: not relevant study $(n=14)$, age $<18$ years $(n=10)$, no information on age ( $n=2)$, participants were foreign immigrants $(n=1)$, unavailability of full-text $(n=1)$. Finally, 22 studies were included in our study for the final in-depth analysis.

The summary of the characteristics of selected studies is presented in Table 1. It includes 18 quantitative studies [10-27] and 4 qualitative studies [28-31]; among the quantitative studies, 5 studies report comparisons with South Korean people [11, 13-15, 22]. The number of participants in these studies varied from 8 to 932 participants, and $54.6 \%-100 \%$ of them were women. The survey using questionnaires was the most popular research method. Data from objective measurement such as body weight, waist circumference, blood test, and urine analysis were used in a few studies [12-14, 19]

Table 1. Summary of the selected studies 


\begin{tabular}{|c|c|c|c|c|}
\hline $\begin{array}{l}\text { Selected } \\
\text { studies }\end{array}$ & Study subject & Study design & Purpose & Measures \\
\hline \multicolumn{5}{|c|}{ Quantitative studies } \\
\hline $\begin{array}{l}\text { Kim et al. (2018) } \\
{[13]}\end{array}$ & $\begin{array}{l}\mathrm{NKD} \\
\mathrm{n}=932 \\
\mathrm{M}, 192 ; \mathrm{W}, 740 \\
\text { Age, } 43.8 \pm 12.6 \text { years } \\
\text { SKN, } \mathrm{n}=1834\end{array}$ & $\begin{array}{l}\text { Cross-sectional } \\
\text { survey }\end{array}$ & $\begin{array}{l}\text { To investigate and compare changes in mean } \\
\text { BMI, WC and prevalence of general and } \\
\text { central obesity among NKRs in SK with } \\
\text { SKN } \\
\text { To investigate factors associated with } \\
\text { general and central obesity among NK }\end{array}$ & $\begin{array}{l}\text { Health-related lifestyle factors: smoking, alcohol } \\
\text { consumption, exercise } \\
\text { WC, body weight }\end{array}$ \\
\hline $\begin{array}{l}\text { Song \& Choi } \\
(2018)[14]\end{array}$ & $\begin{array}{l}\text { NKD } \\
\mathrm{n}=18 \\
\text { Age, } 41.0 \pm 10.0 \text { years } \\
\text { SKN, } \mathrm{n}=472\end{array}$ & $\begin{array}{l}\text { Descriptive } \\
\text { study }\end{array}$ & $\begin{array}{l}\text { To compare the prevalence of NCD and CVD } \\
\text { risk factors between NKR and SK }\end{array}$ & $\begin{array}{l}\text { Questionnaires: Smoking status, alcohol intake, } \\
\text { physical activity, history of medications for diabetes, } \\
\text { hypertension, hyperlipidemia, weight gain, length of } \\
\text { residence } \\
\text { Weight, blood pressure, fasting blood glucose levels, } \\
\text { kidney function }\end{array}$ \\
\hline $\begin{array}{l}\text { Kim et al. } \\
(2016)[11]\end{array}$ & $\begin{array}{l}\text { NKR, } \mathrm{n}=708 \\
\mathrm{M}, 161 ; \mathrm{W}, 547 \\
\text { Age; } 46.9 \pm 12.1 \text { years } \\
(\mathrm{M}) ; 44.1 \pm 11.0 \text { years } \\
\text { (W) } \\
\text { SKN, } \mathrm{n}=1,416\end{array}$ & $\begin{array}{l}\text { Cross-sectional } \\
\text { survey }\end{array}$ & $\begin{array}{l}\text { To determine the prevalence of metabolic } \\
\text { syndrome and its related factors among } \\
\text { NKR in SK population } \\
\text { To compare the prevalence with that in the } \\
\text { general SK population }\end{array}$ & $\begin{array}{l}\text { Disease history } \\
\text { Health-related lifestyle factors: smoking, alcohol, } \\
\text { exercise }\end{array}$ \\
\hline $\begin{array}{l}\text { Park et al. } \\
(2016)[15]\end{array}$ & $\begin{array}{l}\text { North Korean women } \\
\text { who received a Pap } \\
\text { smear within the last } 2 \\
\text { years } \\
\mathrm{n}=385 \\
\text { Age, } 42.92 \pm 8.78 \text { years } \\
\text { SKN, } \mathrm{n}=770\end{array}$ & $\begin{array}{l}\text { Cross-sectional } \\
\text { survey }\end{array}$ & $\begin{array}{l}\text { To identity the rate of cervical cancer } \\
\text { screening of NKD and compare the rates } \\
\text { with South Korean natives } \\
\text { To examine factors associated with cervical } \\
\text { cancer screening among NKD }\end{array}$ & Cervical cancer screening questions \\
\hline $\begin{array}{l}\text { Hyun et al. } \\
\text { (2015) [16] }\end{array}$ & $\begin{array}{l}\text { North Korean immigrant } \\
\text { women } \\
\mathrm{n}=103 \\
\text { Age, } 36.4 \pm 11.4 \text { years }\end{array}$ & $\begin{array}{l}\text { Cross-sectional } \\
\text { survey }\end{array}$ & $\begin{array}{l}\text { To identity the practice of BSE } \\
\text { To examine predictors related to the practice } \\
\text { of BSE among NK female immigrants }\end{array}$ & $\begin{array}{l}\text { BSE practice } \\
\text { Education about BSE and breast cancer screening }\end{array}$ \\
\hline $\begin{array}{l}\text { Wang et al. } \\
(2014) \text { [17] }\end{array}$ & $\begin{array}{l}\mathrm{NKD} \\
\mathrm{n}=498 \\
\mathrm{M}, 101 ; \mathrm{W}, 397 \\
\text { Age, } 35.8 \pm 8.3 \text { years }\end{array}$ & $\begin{array}{l}\text { Cross-sectional } \\
\text { survey }\end{array}$ & $\begin{array}{l}\text { To investigate the self-rated health status of } \\
\text { NKD who have lived in SK for a specific } \\
\text { period of time } \\
\text { To identify the factors related to their self- } \\
\text { rated health status }\end{array}$ & $\begin{array}{l}\text { Special characteristics: duration of residence in South } \\
\text { Korea, satisfaction with support from South Korea } \\
\text { government, satisfaction with current life, traumatic } \\
\text { experiences in North Korea/during escape } \\
\text { Health-related factors: disability status, chronic } \\
\text { diseases, drinking habits }\end{array}$ \\
\hline $\begin{array}{l}\text { Kang et al. } \\
(2012) \text { [18] }\end{array}$ & $\begin{array}{l}\text { NKI } \\
n=96 \\
M, 16 ; W, 80\end{array}$ & $\begin{array}{l}\text { Cross-sectional } \\
\text { survey }\end{array}$ & $\begin{array}{l}\text { To identify the relationships between health } \\
\text { status including objective health status and } \\
\text { subjective health perception HPLP II }\end{array}$ & $\begin{array}{l}\text { Subjective health perception } \\
\text { HPLP-II: responsibility for health, exercise, diet, } \\
\text { spiritual growth, interpersonal relationship, stress } \\
\text { management }\end{array}$ \\
\hline Kim (2009) [19] & $\begin{array}{l}\text { NKR } \\
\mathrm{n}=61 \\
\mathrm{M}, 20 ; \mathrm{W}, 41 \\
\text { Age, } 38.5 \text { years }\end{array}$ & $\begin{array}{l}\text { Descriptive } \\
\text { study }\end{array}$ & $\begin{array}{l}\text { To identify subjectively perceived health } \\
\text { status, health problem, food } \\
\text { intake, exercise, smoking, drinking, stress } \\
\text { and social support }\end{array}$ & $\begin{array}{l}\text { Questionnaires developed by authors: health status, } \\
\text { cancer screening, diet, exercise, smoking, drinking, } \\
\text { stress, social support } \\
\text { Nicotine dependency [18] } \\
\text { Alcohol addiction } \\
\text { Stress checklist } \\
\text { Interpersonal support evaluation }\end{array}$ \\
\hline $\begin{array}{l}\text { Choe et al. } \\
(2012) \text { [20] }\end{array}$ & $\begin{array}{l}\text { NKD } \\
\mathrm{n}=410 \\
\mathrm{M}, 104 ; \mathrm{W}, 306 \\
\text { Age, } 50.8 \pm 0.79 \text { years }\end{array}$ & $\begin{array}{l}\text { Descriptive } \\
\text { study }\end{array}$ & $\begin{array}{l}\text { To identify health knowledge, } \\
\text { health-promoting behavior and factors } \\
\text { influencing health-promoting behavior }\end{array}$ & $\begin{array}{l}\text { Health knowledge } \\
\text { HPLP-II } \\
\text { Perceived health status } \\
\text { Perceived barriers to health-promoting behavior } \\
\text { Self-efficacy: responsibility for health, exercise, diet, } \\
\text { self-belief, interpersonal relationship, stress } \\
\text { management } \\
\text { Social support: professionals, family, friends }\end{array}$ \\
\hline $\begin{array}{l}\text { Jeon \& Park } \\
(2012)[21]\end{array}$ & $\begin{array}{l}\mathrm{NKR} \\
\mathrm{n}=304 \\
\mathrm{M}, 99 ; \mathrm{W}, 205 \\
\text { Age, } 38 \text { years }\end{array}$ & $\begin{array}{l}\text { Descriptive } \\
\text { study }\end{array}$ & $\begin{array}{l}\text { To identify health beliefs and health behavior } \\
\text { To identify factors associated with health } \\
\text { behavior }\end{array}$ & $\begin{array}{l}\text { Health belief } \\
\text { HPLP }\end{array}$ \\
\hline $\begin{array}{l}\text { Yoon \& Kim } \\
(2005)[22]\end{array}$ & $\begin{array}{l}\text { NKD who have lived in } \\
\text { South Korea for more } \\
\text { than } 6 \text { months } \\
\mathrm{n}=306 \\
\mathrm{M}, 139 ; \mathrm{W}, 167 \\
\text { SKN, } \mathrm{n}=7919\end{array}$ & $\begin{array}{l}\text { Descriptive } \\
\text { survey }\end{array}$ & $\begin{array}{l}\text { To examine the health condition, disease } \\
\text { morbidity, and medical service utilization in } \\
\text { SK }\end{array}$ & $\begin{array}{l}\text { Perceived health status } \\
\text { Disease morbidity } \\
\text { Medical service utilization }\end{array}$ \\
\hline $\begin{array}{l}\text { Kim et al. } \\
(2016) \text { [23] }\end{array}$ & $\begin{array}{l}\text { Male NKR } \\
\mathrm{n}=272 \\
\text { Age, } 35.9 \pm 11.3 \text { years }\end{array}$ & $\begin{array}{l}\text { Retrospective \& } \\
\text { descriptive } \\
\text { study }\end{array}$ & $\begin{array}{l}\text { To evaluate the smoking pattern and nicotine } \\
\text { dependence } \\
\text { To identify psychological and psychosocial } \\
\text { states associated smoking }\end{array}$ & $\begin{array}{l}\text { Smoking history } \\
\text { Smoking cessation history } \\
\text { Nicotine dependence (Fagerström test) } \\
\text { Social nicotine dependence (Kano test, KTSND) }\end{array}$ \\
\hline $\begin{array}{l}\text { Jeong et al. } \\
(2017)[12]\end{array}$ & $\begin{array}{l}\text { NKR } \\
\mathrm{n}=149 \\
\mathrm{M}, 26 ; \mathrm{W}, 123 \\
\text { Age, } 48.5 \pm 12.1 \text { years }\end{array}$ & Cohort study & $\begin{array}{l}\text { To understand changes in body weight and } \\
\text { food security over relocation, and current } \\
\text { food and nutrient consumption in SK }\end{array}$ & $\begin{array}{l}\text { Body weight } \\
\text { Food security } \\
\text { Diet: dietary habits, food consumption, energy and } \\
\quad \text { nutrient intake }\end{array}$ \\
\hline $\begin{array}{l}\text { Jeon et al. } \\
\text { (2018) [24] }\end{array}$ & $\begin{array}{l}\text { Female NKD } \\
\mathrm{n}=131 \\
\text { Age, } 37.86 \pm 10.18 \text { years }\end{array}$ & $\begin{array}{l}\text { Cross-sectional } \\
\text { survey }\end{array}$ & $\begin{array}{l}\text { To examine sexual knowledge, sexual } \\
\text { attitude, stress coping and resilience, and } \\
\text { their related factors }\end{array}$ & $\begin{array}{l}\text { Sexual knowledge } \\
\text { Sexual attitude } \\
\text { Stress coping } \\
\text { Resilience }\end{array}$ \\
\hline $\begin{array}{l}\text { Lee \& Shin } \\
(2018)[25]\end{array}$ & $\begin{array}{l}\text { Female NKD } \\
\mathrm{n}=61 \\
\text { Age, } 25.7 \pm 4.0 \text { years }\end{array}$ & $\begin{array}{l}\text { One group } \\
\text { pre/post } \\
\text { design }\end{array}$ & $\begin{array}{l}\text { To evaluate the impact of a mobile video } \\
\text { program on NKD women's health } \\
\text { behavioral change }\end{array}$ & $\begin{array}{l}\text { Knowledge of vaginitis and cervical cancer } \\
\text { Behavioral confidence on prevention and management } \\
\text { for vaginitis and cervical cancer }\end{array}$ \\
\hline $\begin{array}{l}\text { Jeon\& Lee } \\
(2018)[26]\end{array}$ & $\begin{array}{l}\text { NKD } \\
\mathrm{n}=129 \\
\mathrm{M}, 40 ; \mathrm{W}, 89\end{array}$ & $\begin{array}{l}\text { Nonequivalent } \\
\text { control-group } \\
\text { pre-post test } \\
\text { design }\end{array}$ & $\begin{array}{l}\text { To evaluate the customized oral health } \\
\text { promotion program }\end{array}$ & PRECEDE-PROCEED model \\
\hline $\begin{array}{l}\text { Song et al. } \\
\text { (2018) [10] }\end{array}$ & $\begin{array}{l}\text { NKD } \\
\mathrm{n}=399 \\
\text { Age, } 41.0 \pm 10.0 \text { years }\end{array}$ & $\begin{array}{l}\text { Cross-sectional } \\
\text { survey }\end{array}$ & $\begin{array}{l}\text { To determine the association between health } \\
\text { literacy and use of preventive healthcare } \\
\text { services }\end{array}$ & $\begin{array}{l}\text { Health literacy } \\
\text { Influenza vaccination coverage } \\
\text { Medical check-up rates }\end{array}$ \\
\hline Um et al. (2018) & NKR & Cross-sectional & To investigate the determinants of NKR' & Basic communication \\
\hline
\end{tabular}

Page 4/13 


\begin{tabular}{|c|c|c|c|c|}
\hline 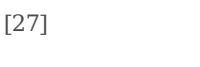 & $\begin{array}{l}\mathrm{n}=168 \\
\mathrm{M}, 49 ; \mathrm{W}, 115\end{array}$ & survey & trust in SK health service & $\begin{array}{l}\text { Health communication } \\
\text { Experience of health service }\end{array}$ \\
\hline \multicolumn{5}{|c|}{ Qualitative studies } \\
\hline $\begin{array}{l}\text { Kim et al. } \\
(2017) \text { [28] }\end{array}$ & $\begin{array}{l}\text { NKR who had lived in } \\
\text { South Korea for more } \\
\text { than } 1 \text { year } \\
\mathrm{n}=8 \\
\text { All women } \\
\text { Age, } 49.8 \pm 11.9 \text { years }\end{array}$ & $\begin{array}{l}\text { Qualitative } \\
\text { study }\end{array}$ & $\begin{array}{l}\text { To understand factors influencing Pap test } \\
\text { use }\end{array}$ & Semi-conducted interview \\
\hline Hong(2015) [29] & $\begin{array}{l}\mathrm{NKD} \\
\mathrm{n}=17 \\
\mathrm{M}, 4 ; \mathrm{W}, 13 \\
\text { South medical providers, } \\
\mathrm{n}=12\end{array}$ & $\begin{array}{l}\text { Qualitative } \\
\text { study }\end{array}$ & $\begin{array}{l}\text { To investigate the differences between the } \\
\text { views of NKD and their medical providers } \\
\text { in regard to the prescription non- } \\
\text { adherence and the causes of those } \\
\text { differences }\end{array}$ & $\begin{array}{l}\text { Three group interviews } \\
\text { Individual interview }\end{array}$ \\
\hline $\begin{array}{l}\text { Choi \& Choi } \\
\text { (2009) [30] }\end{array}$ & $\begin{array}{l}\text { Saetomins who have } \\
\text { visited a hospital with at } \\
\text { least one chronic } \\
\text { disease in South Korea } \\
\mathrm{n}=11 \\
\mathrm{M}, 3 ; \mathrm{W}, 8 \\
\text { Age, } 60.1 \pm 8.1 \text { years }\end{array}$ & $\begin{array}{l}\text { Qualitative } \\
\text { study }\end{array}$ & To explore treatment-seeking behavior & Semi-conducted interview \\
\hline $\begin{array}{l}\text { Chung et al. } \\
(2018)[31]\end{array}$ & $\begin{array}{l}\text { Women NKD } \\
\mathrm{n}=10 \\
\text { Age, } 55-73 \text { years }\end{array}$ & $\begin{array}{l}\text { Qualitative } \\
\text { study }\end{array}$ & $\begin{array}{l}\text { To explore and describe the health-seeking } \\
\text { experience of women NKD }\end{array}$ & In-depth, unstructured interview \\
\hline
\end{tabular}

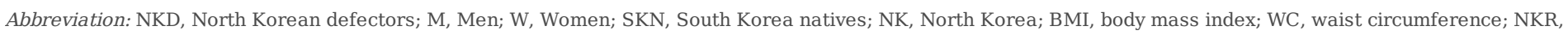

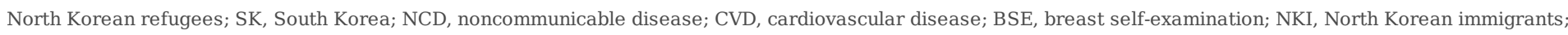
HPLP, Health Promoting Lifestyle Profile; KTSND, Kano test for social nicotine dependence

Table 2 shows the variables that were investigated in the selected studies by classifying them into 4 categories: non-communicable disease, health promotional lifestyle, self-rated health status, and others.

Table 2. Results of selected quantitative studies 


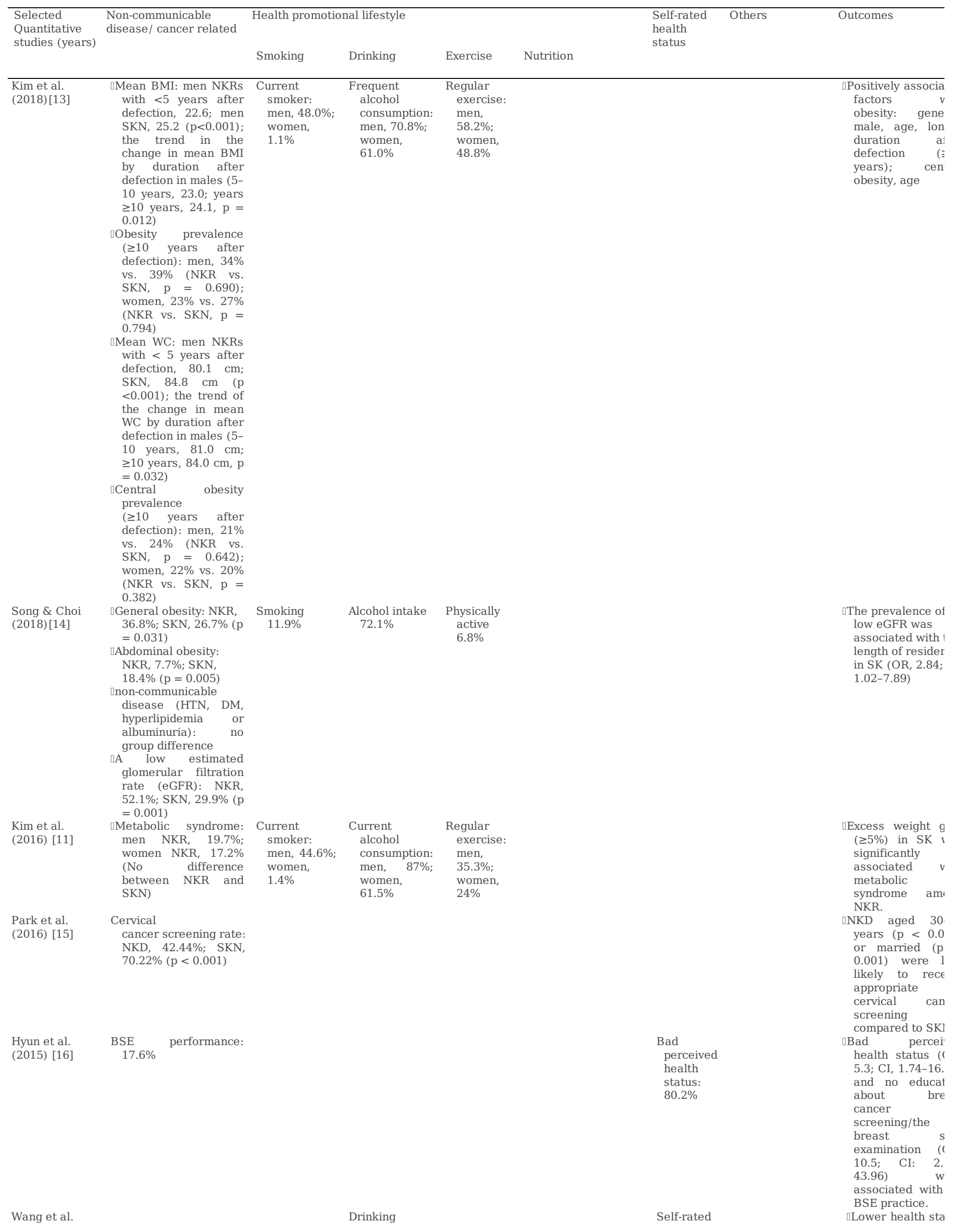


pattern: once or less $/ \mathrm{m}$, $25.5 \%$; twice or more $/ \mathrm{m}$, $31.3 \%$
Kang et al.

(2012) [18]

Kim(2009) [19] घEarly screenings rate of the major 5 cancer: gastric cancer, $31.1 \%$; hepatoma, $27.9 \%$; colorectal cancer,

$13.1 \%$; cervical cancer, $36.6 \%$; breast cancer, $12.2 \%$

Choe et al.

(2012) [20]

Yoon \&

$\operatorname{Kim}(2005)$ [22]

$\begin{array}{lll}\begin{array}{c}\text { Physical } \\ \text { activity, }\end{array} & \text { Nutrition, } 2.25(1-4) & \text { Current } \\ 1.71 \quad(1- & & \text { poor } \\ 4) & \text { health } \\ & & \text { status, } \\ & 40 \%\end{array}$

Current
smoking
status,
$26.2 \%$;
nicotine
dependency,
$21.3 \%$

Current

status,

$37.7 \%$

$21.3 \%$

status: $42.7 \%$

$\begin{array}{ccccc}\text { Non-smoking, } & \text { Reducing } & \text { Exercise, } & \text { Dietary habit, } 3.0 \pm \\ 2.9 \pm 0.72 & \text { alcohol, 2.8 } & 2.6 & \pm & 0.5(1-4) \\ (1-4) & \pm 0.66(1-4) & 0.68 \quad(1- & & \end{array}$

health:

$2.78 \pm$

1.14 out

of 5 was related $v$ women $(\mathrm{p}<0.0$ ( elderly $(\mathrm{p}<0.0$ ( or had low ann household inco ( $\mathrm{p}=0.017)$, ; chronic diseases $<0.001$ ).

口Higher self-ra health status I associated v settlement in for 18 months more $(p=0.02$ satisfaction government support $(p=0.0$ or their current

$(\mathrm{p}=0.001)$, ; more traum events experie: in North Korea ( $0.039)\left(\mathrm{R}^{2}=0.0\right.$ $\mathrm{p}<0.001)$

HPLP-II: $1.78 \pm$ Women $(\mathrm{p}=0.01$ $0.35(1-4) \quad$ normal weight overweight ( $p$ 0.006)

inhabitation over 1 year South Korea (p 0.026) were rela to the lower sc of HPLP II.

पStress: $8.56 \pm$ $5.08(0-24)$

\Social support: $24.20 \pm 14.90$ $(0-54)$

Perceived

health status: mean,

4.16

10)

(1- 口HPLP-II: $2.38 \pm$ 0.57 (1-4)

पHealth beliefs: $2.8 \pm 0.29$ (14) 口HPLP: $2.9 \pm 0.38$ (1-4)

Factors influenc
Subjective evaluated worse health condition: NKD, $35.2 \%$;

SKN, $12.6 \%$
aDisease

morbidity NKD, 75.5\%; SKN, 46\% $\square$ Medical service utilization: NKD, $\quad 64.8 \%$; SKN, 87.6\%;
Kim et al.

(2016) [23]

\footnotetext{
๑Current

smokers,

$84.2 \%$

$\square$ Smoking

age, $18.2 \pm$

4.7 years

口Fagerström

test, $3.35 \pm$

2.26 (0-10);
} health-promotins behavior of participants $\mathrm{W}$ found to be $s$ efficacy ( $p$ 0.001), so support $(\mathrm{p}<0.0$ and percei barrier health-promotins behavior ( $\mathrm{p}$ $0.001)\left(R^{2}=0.0\right.$ $\mathrm{p}<0.001)$.

The fact influencing health behavior NK

refugees: percei benefits ( $p$ 0.001), $\mathrm{s}$ efficacy (p $0.001)$, the per in the $t$ l country

0.031)

experience in be expelled to NK ( 0.010) 
KTSND

$13.76 \pm 4.87$

Jeong et al.

Food security status: from $12.1 \%$ to $61.7 \%$

aLess food security in SK appeared in the body weight loss group than the other two

(maintenance/gain) groups ( $p=0.02$ ).

The body weight loss group showed the most irregular meal consumption pattern $(\mathrm{p}<0.05)$.

Jeon et a

(2018) [24]

Lee \& Shin (2018) [25]

Jeon\& Lee (2018) [26]

Song et al. (2018) [10]

\section{Perceived health \\ status,}

$3.66 \pm$

$0.66(1-5)$

$\square$ Sexual
knowledge,0.58
$\pm 0.11(0-1)$
$\square$ Sexual attitude,
$2.88 \pm 0.36(1-$
$5)$
$\square$ Stress coping,
$1.41 \pm 0.50(0-$
$3)$
$\square$ Resilience, 3.81
$\pm 0.72(1-5)$

$\pm 0.72(1-5)$

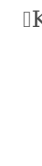

vaccinatio

$31.1 \%$

Medical check-

up, $58.5 \%$

(within 2

years) $\square$ Oral health belief $=0.004$ ) and ora health knowledg were significant improved $(\mathrm{p}=$ 0.003) in experimental grc 口Oral health behaviors in experimental grc was significantly improved compa with controls (p 0.05 )

etter literacy sco were more likely have vaccinat (adjusted $\mathrm{OR}=2$. 95\%CI, 1.19-5.0 In subgroup analysis, NKD w lived alone $(\mathrm{p}=$ 0.032 ), longer ti in other countrie before entering $=0.007$ )

The determinants trust in SK he services: durat of residence ( $\mathrm{F}$ 0.01 ), communication skills ( $\mathrm{p}<0.0$ ( health communication skills $(\mathrm{p}<0 . \mathrm{C}$ 


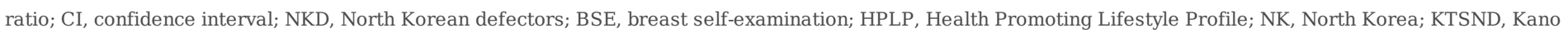
test for social nicotine dependence

\section{NCD-related studies}

Three studies reported the prevalence of obesity and metabolic diseases. In comparison with South Korean natives (SKN), one study showed that the obesity and central obesity prevalence rate in North Korean refugee (NKR) men increased by duration after defection, and all the prevalence rates were comparable to those of SKN for both the genders after $>10$ years of defection (obesity prevalence NKR vs. SKN: men, $34 \%$ vs. $39 \%, p=0.690 ;$ women, $23 \%$ vs. $27 \%, p=0.794$; central obesity, $21 \%$ vs. $24 \%, p=0.642$; and women, $22 \%$ vs. $20 \%, p=0.382$, respectively) [13]. Our results are similar with another study wherein the excess weight gain after defection was associated with metabolic syndrome with similar prevalence rate of metabolic syndrome between NKR and SKN (men, $19.7 \%$ vs. $26.2 \%, p=0.134$; and women, $17.2 \%$ vs. $16.6 \%, p=0.757$, respectively) [11]. Another study showed no group difference in the prevalence of NCDs, such as hypertension, diabetes mellitus, and hyperlipidemia; however, general obesity of NKR was higher (NKR vs. SKN, $36.8 \%$ vs. $26.7 \%, p=0.031$, respectively) and abdominal obesity of NKR was lower than that of South Korean counterparts (NKR vs. SKN, 7.7\% vs. 18.4\%, $p=0.005$, respectively) [14].

\section{Health promotional lifestyle studies}

The health behaviors of North Korean defectors are summarized in terms of smoking, drinking, exercise, and nutrition in Table 2. The rate of current smokers among NKRs was $11.9 \%$ [14], 26.2\% [19], and 84.2\% [23]. The rate of current smokers in men was $48.0 \%$ [13] and $44.6 \%$ [11]. One study reported that 21.3\% of the smokers were nicotine dependent [19], and the other study demonstrated low to moderate dependent status on nicotine with a score of 3.35 out of 10 (Fagerström test for nicotine dependence) [23]. Moreover, the scores of health behavior for non-smoking and preventive behavior for smoking were 2.9 out of 4 (health-promoting lifestyle profile, HPLP) [21].

The current drinkers comprised of 72.1\% [14] and 37.7\% [19].Two studies reported that the current drinkers among men were 70.8\% [13] and 87\% [11].The rate of alcohol consumption more than once a month was $56.8 \%$ [17]. The score of health behavior for reducing alcohol consumption was 2.8 (HPLP, range 1-4) [21].

For exercise, $6.8 \%$ [14], 32.8\% [19] , and 58.2\% [13] of the North Korea defectors were reported to exercise regularly, and 35.3\% [11] in men were also reported to do regular exercise. The score of health behavior for exercise was 1.71 (HPLP-II, range 1-4) [18], 2.23 (HPLP-II, range 1-4) [20], and 2.6 (HPLP, range1-4) [21].

In case of nutrition, $55.7 \%$ had regular meals [19], and food security status, which includes food consumption with sufficient amount and various kinds of food, improved from $12.1 \%$ when staying in North Korea to $61.7 \%$ while staying in South Korea [12]. Less food security status was shown in terms of the body weight loss group, which includes the most irregular meal consumption pattern, than the body weight maintenance or gain group $(p<0.05)$ [12]. The scores of health behavior for dietary habit were 2.25 (HPLP-II, range1-4) [18], 2.44 (HPLP-II, range 1-4) [20], and 3.0 (HPLP, range 1-4) [21].

For cancer screening rate, $42.44 \%$ [15] or $36.6 \%$ [19] of NKRs had a cervical cancer checkup and other major cancers were also screened [19]; gastric cancer, $31.3 \%$; hepatoma, $27.9 \%$; colorectal cancer, $13.1 \%$; and breast cancer, $12.2 \%$.

\section{Self-rated health status studies}

Subjectively reported health conditions were found in 8 studies (Table 2). Among North Korean defectors, 40\% [18], 42.7\% [19], and 74.2\% [27] rated their subjective health status as poor. Even $80.2 \%$ North Korean women reported their health status as poor [16]. Compared with $12.6 \%$ of South Korean natives, $35.2 \%$ of NKRs had poor health status [22]. It is also reported that the mean scores of perceived health status were 2.78 (range 1-5) [17], 4.16 (range 1-10) [20], and 3.66 [25].

\section{Others}

This category included a variety of variables, including sub or total score of HPLP [18, 20, 21], medical service utilization [22], health-related [20] or sex-related knowledge and attitude [24, 25], and vaccination coverage [10].

\section{Qualitative study}

Among the 4 qualitative studies, 1 study investigated factors affecting cervical cancer screening [28], 2 studies for treatment adherence [29, 30], and 1 study for health-seeking experience [31] (Table 3). NKRs commonly had poor or mistaken knowledge about cervical cancer [28] as well as symptoms associated with psychological/socio-cultural influence and the concept of staged treatments [29]. They also had incorrect awareness or mistrust that "cancer is a fatal disease" [28] or "medical environment in South Korea did not offer appropriate treatment for their physical health problems", while they did not know that they were antibiotic-resistant [29]. The NK women defectors also reported a low level of awareness of diseases, not to have known about CVD such as hypertension, hypotension, diabetes or any other disease until their first health check-up after they entered South Korea. Furthermore, they were reported to express discomfort and uneasiness with the unfamiliar South Korean health care system and the economic burden to afford a healthy life under the actbased reimbursement system with the fees charged for diagnosis, treatment, and medication. [31]. 
Table 3. Results of selected qualitative and review studies

\begin{tabular}{|c|c|}
\hline $\begin{array}{l}\text { Selected studies } \\
\text { (years) }\end{array}$ & Outcomes \\
\hline $\begin{array}{l}\text { Kim et al. } \\
(2017)[28]\end{array}$ & $\begin{array}{l}\text { Barrier factors } \\
\text { individual level: lack of knowledge about cervical cancer and Pap test, cancer worry, unfamiliar with receiving preventive care and concerns } \\
\text { about cost } \\
\text { community level: negative health outlook such as viewing cancers as fatal diseases } \\
\square \text { Facilitator factors } \\
\text { interpersonal level: social support from family, female healthcare providers } \\
\text { systemic level: free screening programs }\end{array}$ \\
\hline Hong(2015) [29] & $\begin{array}{l}\text { The cause of prescription non-adherence: } \\
\text { physical symptoms vs. psychological/socio-cultural influences } \\
\text { trust in self-diagnosis and established beliefs vs. suspicion caused by this trust and these beliefs } \\
\text { lack of medical treatments appropriate for NKD vs. the presence of tolerant bacterial strains } \\
\text { slow and ineffective due to capitalism vs. lack of understanding the concept of staged treatments }\end{array}$ \\
\hline $\begin{array}{l}\text { Choi \& Choi } \\
\text { (2009) [30] }\end{array}$ & $\begin{array}{l}\text { घNew experience related to treatment: physical abnormalities that were discovered after arriving in South Korea, an unfamiliar treatment } \\
\text { environment, the cost and the benefit of the medical treatment, and an increased concern about one's improving health } \\
\text { 口Types of treatment seeking behavior: being compliant with medical care, managing symptoms with self-treatment, and seeking complementary } \\
\text { and alternative treatment }\end{array}$ \\
\hline $\begin{array}{l}\text { Chung et al. } \\
\text { (2018) [31] }\end{array}$ & $\begin{array}{l}\text { The } 4 \text { categories of health-seeking experience: finding out about my own body, confusion regarding the medical treatment, and enjoying the health } \\
\text { care benefits, and protecting my own health }\end{array}$ \\
\hline
\end{tabular}

Abbreviation: NKD, North Korean defectors

\section{Discussion}

As far as we know, this is the first systematic review of the health-promoting behaviors of North Korean defectors, which can indirectly represent North Koreans. In this study, we showed that there were not enough studies related to NCDs and health behaviors of North Korean defectors. Nevertheless, North Korean defectors were found to be more vulnerable to NCD diseases, including metabolic syndrome, owing to higher smoking rates, drinking rates, and increased abdominal obesity caused by lack of awareness and management of risk factors since they moved to South Korea.

Owing to the nature of the closed and centrally controlled North Korean society, it is difficult to obtain secure reliable data on the health status of North Koreans or related factors. Although the rate of smoking and alcohol drinking of North Korean defectors varied among the studies [11, 13, 14], we have confirmed that there have been significant differences between the internationally reported smoking rate and drinking rate among North Koreans and those of North Korean defectors. According to a recent report on North Korean defectors in Hanawon (The Settlement Support Center for North Korean Refugees) where North Korean defectors lived for the first 3 months of settlement in South Korea, $84.2 \%$ of men were reported as current smokers, $12.5 \%$ as ex-smokers, and $3.3 \%$ as non-smokers [23], which is quite different from the North Koreans' smoking rate reported in the Tobacco Atlas in 2015 (37.3\% smoking rate in men) and the World Health Organization's (WHO) report (46.1\% smoking rate in men) [32, 33]. In addition, about $60 \%-80 \%$ of North Korean defectors reported frequent or daily alcohol consumption $[11,13,14]$. According to the WHO 2018 report, the average daily intake of pure alcohol in North Korea was $20.7 \mathrm{~g}$ (South Korea $34.6 \mathrm{~g}$ of pure alcohol), and the rates of heavy drinking episodes were $23.7 \%$ in men and $3.9 \%$ in women in North Korea, which were lower compared to $47.8 \%$ in men and $13.6 \%$ in women in South Korea [34-36].

In the comparative study about physical activity, different results for North Korea defectors and South Koreans were reported (physically active, North Korean defector $8 \%$ vs South Korean $67 \%$ [14], regular exercise in men $35.3 \%$, women $24.0 \%$ among North Korean defector vs men $24.2 \%$, women $18.5 \%$ among South Korean) [11]. Unbalanced nutrition intake was reported from $98.4 \%$ for North Korean defectors along with relatively low intake of protein, vitamins, and calcium [19]. Currently, with doubts regarding the reliability of health data generated in North Korea, the health data of North Korean defectors are important because the data can be indirectly represented for North Koreans.

We reviewed the best available data for demonstrating the North Korean defectors' health behaviors related to NCD in this study. North Korean defectors are reported to have a negative perception on their health status compared to South Koreans, and low literacy and knowledge of health have had a negative impact on their health behavior $[10,28,29]$.

Altogether, North Korean defectors seemed to be more vulnerable to obesity and metabolic syndrome during settlement in South Korea, which are the major risk factors of CVD. Moreover, extreme stress during the process of immigration and settlement in South Korea may exacerbate North Korean defectors' unhealthy behavior [21]. These results are consistent with the results of previous studies that diabetes and cardiovascular disease are more prone to occur when defectors from poor countries move to developed countries owing to rapid lifestyle changes and more stress under the new environment [37, 38]. Overall, understanding the socio-cultural influences on North Korea defectors in advance is necessary for the development and implementation of tailored-health programs for North Korean defectors [30,31]. It is essential to improve the awareness of health behaviors and disease status on CVD among North Koreans defectors. However, a recent review showed that the majority of NCD studies on North Korean defectors addressed mental health issue [39].

Establishing a systematic education program could dispel the misunderstandings and misperceptions related to health, which originated from cultural and social differences $[10,29]$. South Korean government and the health care professionals should make efforts for preventing and intervening at an early stage of NCD diseases among North Korean defectors by sharing health data of all the North Korean defectors. However, since there were a limited number of participants (61-982) in the reviewed literature for this study, it seems necessary to disclose and share health-related data of all the North Korean defectors who were already established by the government for research purposes and for establishing an evidence-based clinical practice strategy.

There are several limitations to this study. First, the reviewed literature about North Korean defectors included mainly women and young adult group; therefore, a biased population might not be a representative sample of North Koreans, and hence, careful interpretation was needed. Second, the data from objective measurements, such as anthropometric or biochemical analysis, were included in a few studies. Third, North Korean defectors had different residence periods

Page $10 / 13$ 
in South Korea in each study. As the settlement period of migrants and the occurrence of NCD are closely associated, it is necessary to conduct large-scale cohort research on the health status of North Korean defectors to overcome these limitations.

\section{Conclusions}

We reviewed the best available data for demonstrating North Korean defectors' health-promoting behaviors related to NCD. It is necessary to improve the awareness of NCD and its risk factors among North Korean defectors. Efforts for promoting North Korean defectors' health behaviors by the government and related professional organizations are needed. Developing a strategy for promoting North Korean defectors' health awareness and behavior, preventing or controlling NCD, and evaluating its effectiveness could further contribute to developing a healthcare delivery model, which is applicable to North Korea in the near future.

\section{List Of Abbreviations}

NKD, North Korean defectors; M, Men; W, Women; SKN, South Korea natives; NK, North Korea; BMI, body mass index; WC, waist circumference; NKR, North Korean refugees; SK, South Korea; NCD, noncommunicable disease; CVD, crdiovascular disease; BSE, breast self-examination; NKI, North Korean immigrants; HPLP, Health Promoting Lifestyle Profile; KTSND, Kano test for social nicotine dependence; eGFR, estimated glomerular filtration rate; OR, odds ratio; Cl, confidence interval; BSE, breast self-examination; KTSND, Kano test for social nicotine dependence

\section{Declarations}

\section{Acknowledgments: None}

Funding: This work was supported by Yonsei University College of Nursing (6-2018-0135).

Authors' contributions: HYS contributed to the study design, analysis, and an initial draft of introduction and discussion. JES directed the study design, data collection, analysis, and contributed to an initial draft of methods and results. SYB assisted with the data collection and analysis and created a figure and tables. SHC contributed to the study design, analysis, and revisions to the article.

Ethics approval and consent to participate: Not required

Consent for publication: Not applicable

Competing interests: None applicable

Data sharing statement: No additional data are available

\section{References}

1. Lee YH, Yoon SJ, Kim YA, Yeom JW, Oh IH. Overview of the burden of diseases in North Korea. J Prev Med Public Health. 2013;46(3):111-7.

2. Ezzati M, Pearson-Stuttard J, Bennett JE, Mathers CD. Acting on non-communicable diseases in low- and middle-income tropical countries. Nature. 2018;559(7715):507-16.

3. Choi J. An Analysis of demographic structural change in North Korea and its implications. North Korean Stud Rev. 2016;20(2):1-30.

4. Grundy J, Moodie R. An approach to health system strengthening in the Democratic Peoples Republic of Korea (North Korea). Int J Health Plann Manage. 2009;24(2):113-29.

5. Grundy J, Biggs BA, Hipgrave DB. Public Health and International Partnerships in the Democratic People's Republic of Korea. PLoS Med. 2015;12(12):e1001929.

6. WHO Vaccine-preventable disease: monitoring system. 2013 global summary (Democratic People's Republic of Korea). https://apps.who.int/immunization_monitoring/globalsummary/countries?countrycriteria\%5Bcountry\%5D\%5B\%5D=PRK Accessed 14 July 2019.

7. Hwang NM. Strategies and tasks in healthcare for Korean reunification. In: Issue \& Focus. Korea Institute for Health and Social Affairs; 2014;240:1-8.

8. Institute for Health Metrics and Evaulation. http://www.healthdata.org Accessed 14 July 2019.

9. WHO. Noncommunicable disease. http://www.who.int/mediacentre/factsheets/fs355/en Accessed 14 July 2019.

10. Song IG, Lee H, Yi J, Kim MS, Kawachi I, Park SM. Health literacy and use of preventive health services among North Korean defectors in the Republic of Korea. PLoS One. 2018; 13(6):e0195964.

11. Kim YJ, Lee YH, Lee YJ, Kim KJ, An JH, Kim NH, Kim HY, Choi DS, Kim SG. Prevalence of metabolic syndrome and its related factors among North Korean refugees in South Korea: a cross-sectional study. BMJ Open. 2016;6(6):e010849.

12. Jeong H, Lee SK, Kim SG. Changes in body weight and food security of adult North Korean refugees living in South Korea. Nutr Res Pract. 2017;11(4):30718.

13. Kim YJ, Kim SG, Lee YH. Prevalence of general and central obesity and associated factors among North Korean fefugees in South Korea by duration after defection from North Korea: a cross-sectional study. Int J Environ Res Public Health. 2018;15(4):811.

14. Song YS, Choi SW. Low estimated glomerular filtration rate is prevalent among North Korean refugees in South Korea. Korean J Fam Med. 2018;39:16167. 
15. Park J, Kim H, Yang W, Lee H, Park SM. Cervical cancer screening and its associated factors among North Korean defectors living in South Korea. J Immigr Minor Health. 2016; 20(1):66-72.

16. Hyun MY, Song HJ, Lee EJ, Hong SC, Kim SY, Lee CH. Predictors of breast self examination practice among North Korean immigrant Women. Korean J Health Promot. 2015;15(1):9-15.

17. Wang B, Yu S, Noh J, Kwon YD. Factors associated with self-rated health among North Korean defectors residing in South Korea. BMC Public Health. 2014;14:999.

18. Kang Y, Ha Y, Eun Y. Health status and health-promoting lifestyle profile II of North Korean immigrants. J Korean Acad Community Health Nurs. 2012;23(3):231-43.

19. Kim Y. A survey on the health status of North Korean refugees in a region. J Korean Soc Emerg Med. 2009;13(1):5-18.

20. Choe MA, Yi M, Choi JA, Shin G. Health knowledge, health promoting behavior and factors influencing health promoting behavior of North Korean defectors in South Korea. J Korean Acad Nurs. 2012;42(5):622-31.

21. Jeon JH, Park Y. The effects of individual characteristics and health beliefs on North Korean refugees' health behavior. J Korean Acad Community Health Nurs. 2012;23(1):82-90.

22. Yoon IJ, Kim S. Health and medical care of North Korean defectors in South Korea. Health and Social Science. 2005;17:149-82.

23. Kim SW, Lee JM, Ban WH, Park CK, Yoon HK, Lee SH. Smoking habits and nicotine dependence of North Korean male defectors. Korean J Intern Med. 2016;31(4):685-93.

24. Jeon JH, Kwon MO, Kim H, Jung Y, Hwang SM, Ha B. Sexual knowledge, sexual attitude, stress coping, and resilience of North Korean female defectors. Journal of Military Nursing Research. 2018;36(1):22-34.

25. Lee MK, Shin G. A mobile video intervention for women's health of North Korean defectors. Public Nealth Nurs. 2018;35:558-62.

26. Jeon K, Lee S. Evaluation of customized oral health promotion program for North Korean defector. J Korean Acad Oral Health. 2018;42(4):109-17.

27. Um TR, Lee TH, Lee EJ, Jin KN. Determinants of North Korean refugees' trust in South Korea health service. Health Policy and Management. 2018;28(1):70-6.

28. Kim K, Kim S, Chung Y. A qualitative study exploring factors associated with Pap test use among North Korean refugees. Health Care Women Int. 2017;38(10):1115-29.

29. Hong SJ. Not at all effective: differences in views on the causes of prescription non-adherence between North Korean defectors and medical providers in South Korea. J Immigr Minor Health. 2015;17(3):867-84.

30. Choe MA, Choi JA. A study on treatment-seeking behavior of middle-aged and old-age saetomins in South Korea. Journal of peace and unification studies. 2009;1(1):285-316.

31. Chung $\mathrm{CH}$, Kang HY. Health-seeking experience of North Korean women defectors in South Korea. Public Nealth Nurs. 2018;35:192-201.

32. The tobacco atlas. https://tobaccoatlas.org/country/dpr-korea Accessed 14 July 2019.

33. WHO. WHO report on the global tobacco epidemic. In: Country profile Democratic People's Republic of Korea. 2019. https://www.who.int/tobacco/surveillance/policy/country_profile/prk.pdf Accessed 14 July 2019.

34. WHO. Management of substance abuse. In: Global status report on alcohol and health 2018. https://www.who.int/substance_abuse/publications/global_alcohol_report/en Accessed 29 July 2019

35. WHO. Alcohol consumption: levels and patterns (Democratic People's Republic of Korea) https://www.who.int/substance_abuse/publications/global_alcohol_report/profiles/prk.pdf?ua=1 Accessed 29 July 2019.

36. WHO. Alcohol consumption: levels and patterns (Republic of Korea) https://www.who.int/substance_abuse/publications/global_alcohol_report/profiles/kor.pdf Accessed 21 Aug 2019.

37. Kaplan MS, Huguet N, Newsom JT, McFarland BH. The association between length of residence and obesity among Hispanic immigrants. Am J Prev Med. 2004;27(4):323-6.

38. Goel MS, McCarthy EP, Phillips RS, Wee CC. Obesity among US immigrant subgroups by duration of residence. JAMA. 2004;292(23):2860-7.

39. Park JJ, Lim AY, Ahn HS, Kim Al, Choi S, Oh DH, Lee-Part O, Kim SY, Jung SJ, Bump JJ, Atun R, Shin HY, Part KB. Systematic review of evidence on public health in the Democratic People's Republic of Korea. BMJ Glob Health. 2019;4(2):e001133.

\section{Figures}




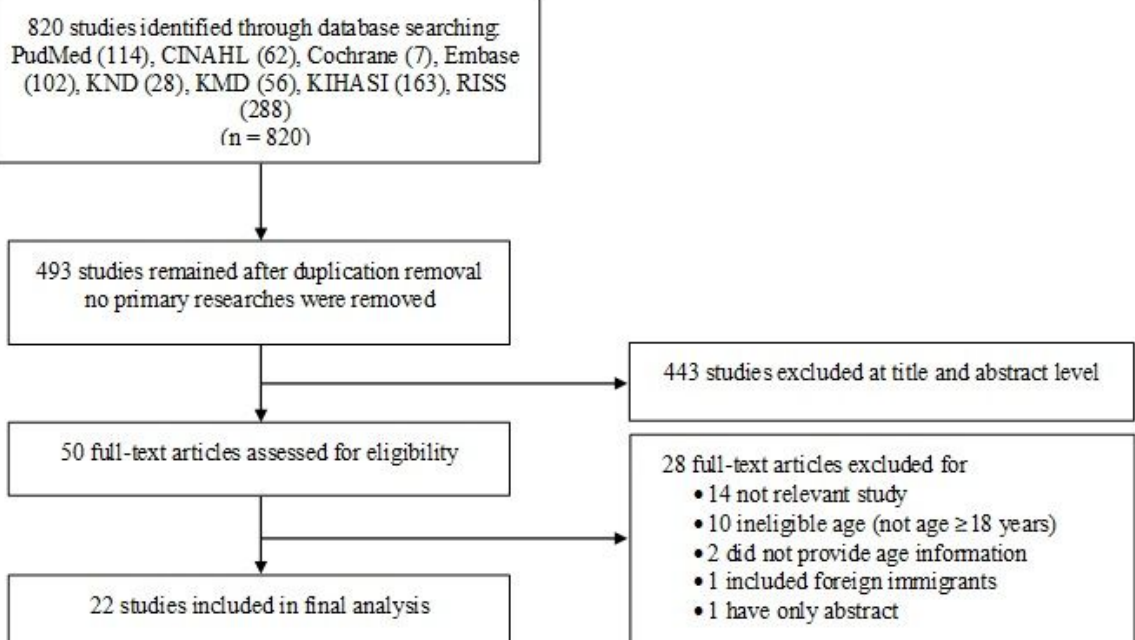

\section{Figure 1}

Literature searching flow diagra 